regression was performed to identify independent predictors of unilateral/bilateral detection.

Results $\mathrm{He}$ mean age and BMI was 66.9 years and $31.8 \mathrm{Kg} /$ $\mathrm{m} 2$, respectively. 90 women $(52.3 \%)$ were diagnosed with endometrioid histology, whilst 82 with other high-risk histology. In total, 321 SLNs were removed, whilst at least one SLN was obtained in 151 women for a detection rate of $87.8 \%$. In 106 women (61.6\%) bilateral SLNs were successfully mapped. On average $1.87(0-5)$ SLNs were detected per patient. SLNs were most commonly identified in the external iliac basins (78.2\%), followed by the obturator fossa (10.3\%), internal iliac basins (5.9\%), common iliac basins $(3.73 \%)$, pre-sacral $(0.93 \%)$ and para-aortic region $(0.93 \%)$, respectively. Lymph node metastasis was detected in 25 women $(14.5 \%)$. There was no statistical correlation between the SLN detection and the age, BMI, grade and histology, respectively. The bilateral SLN detection was adversely correlated with grade 3 (rho $=-0.29, \mathrm{p}$-value $=0.0001$ and high-risk histology $(\mathrm{rho}=-0.3, \mathrm{p}$-value $=0.0001)$. In multivariate analysis, both grade $(\mathrm{OR}=0.21, \mathrm{p}$-value $=0.005)$ and high-risk histology $(\mathrm{OR}=0.39, \mathrm{p}$-value $=0.04)$ remained significant. Only three cases of Grade 1 lower extremity lymphoedema were reported.

Conclusion Intra-operative SLN mapping using fluorescence imaging with ICG in EC patients is feasible, yields high detection rates and reduces the lymphadenectomy-associated morbidity. Further studies are warranted to evaluate its accuracy in high-risk EC.

Disclosures We certify that no party has a direct interest in the results of the research and that no benefit will be conferred to us or any organisation with which we are associated.

\section{DUAL CERVICAL AND FUNDAL INJECTION IN ENDOMETRIAL CANCER}

${ }^{1}$ Mikel Gorostidi, ${ }^{2}$ Ruben Ruiz Sautua, ${ }^{2}$ Ibon Jaunarena, ${ }^{2}$ Paloma Cobas, ${ }^{2}$ Arantxa Lekuona. ${ }^{1}$ Osakidetza, Hospital Universitario Donostia; Gyn Onc; ${ }^{2}$ Osakidetza, Hospital Universitario Donostia

\subsection{6/ijgc-2020-ESGO.83}

Introduction/Background Sentinel node in endometrial cancer (EC) is an evolving technique to know the nodal status in a more precise way without the need to perform a complete lymphadenecotomy. The aim of this study was to describe our latest results using dual cervical and fundal indocyanine green (ICG) injection for detection of sentinel lymph node (SLN) in endometrial cancer and results of SLN biopsy.

Methodology This is an observational prospective study performed between 26 June 2014 and 31 December 2019, 278 patients underwent laparoscopic surgery for endometrial cancer at Hospital Uniersitario Donostia, in Spain. In all cases, we performed SLN biopsy with dual cervical and fundal ICG injection, looking for pelvic and aortic sentinel node. All SLNs were processed with an ultrastaging technique. A total of 128 patients with Intermediate and High risk EC also underwent total pelvic and paraaortic lymphadenectomy.

Results The detection rates were as follows: 93.52\% (260/ $278)$ overall for SLNs; 90.65\% (252/278) overall for pelvic SLNs; 67.99\% (189/278) for bilateral SLNs; 66.91\% (186/ 278) for paraaortic SLNs, and 2.88\% (8/278) for isolated paraaortic SLNs. We found macroscopic lymph node metastasis in 26 patients $(10.03 \%)$ and microdisease in lymph nodes in another 48 patients, raising the overall rate of lymph node involvement to $16.19 \%$. There was one false negative (negative SLN biopsy but positive lymphadenectomy). $6.6 \%$ of all pelvic and also aortic sentinel nodes were positive for metastasis. Applying the $\mathrm{SN}$ algorithm, the sensitivity of detection was $97.9 \%$ (95\% CI 89.1-99.6), specificity $100 \%$ (95\% CI 98.2-100), negative predictive value 99.5\% (95\% CI 97.4-99.9), and positive predictive value 100\% (95\% CI 92.4-100).

Conclusion Dual sentinel node injection is a feasible technique that achieves adequate detection rates. Additionally, this technique allows a high rate of aortic detection, identifying a nonnegligible percentage of isolated aortic metastases. Aortic metastases in endometrial cancer are possible and it is necessary to investigate the relevance of its detection.

Disclosures No disclosures.

\section{Fertility pregnancy}

\section{FERTILITY-SPARING TREATMENT IN ADVANCED BORDERLINE OVARIAN TUMORS. AN ANALYSIS FROM THE MIT014 STUDY DATABASE}

${ }^{1}$ Francesca Falcone, ${ }^{1}$ Stefano Greggi, ${ }^{2}$ Pierandrea De laco, ${ }^{3}$ Gabriella Ferrandina, ${ }^{4}$ Gennaro Cormio, ${ }^{5}$ Violante DI Donato, ${ }^{6}$ Giorgia Mangili, ${ }^{7}$ Francesco Raspagliesi, ${ }^{8}$ Mario Malzoni, ' ${ }^{9}$ Enrico Breda. 'Istituto Nazionale Tumori, Ircss, 'fondazione G. Pascale', Department of Gynecologic Oncology; ${ }^{2}$ Sant'orsola-Malpighi Hospital; Gynecologic Oncology Unit; ${ }^{3}$ Fondazione Policlinico Universitario A. Gemelli, Irccs; Department of Woman and Child Health and Public Health; ${ }^{4}$ University of Bari 'aldo Moro'; Department of Biomedical Sciences and Human Oncology, Unit of Obstetrics and Gynecology; 'Sapienza' University; Department of Maternal and Child Health and Urological Sciences; ${ }^{6}$ Irccs Ospedale San Raffaele; Obstetrics and Gynecology Department; ${ }^{7}$ Fondazione Irccs Istituto Nazionale Dei Tumori; Gynecologic Oncology; ${ }^{8}$ Center for Advanced Endoscopic Gynecologic Surgery; Endoscopica Malzoni; ${ }^{9}$ Ospedale S. Giovanni Calibita Fatebenefratelli; Medical Oncology Unit

\subsection{6/ijgc-2020-ESGO.84}

Introduction/Background For advanced borderline ovarian tumors (BOTs), data concerning the efficacy and safety of fertility-sparing surgery (FSS) are very limited. The MITO14 is a multi-institutional retrospective study conducted among MITO Centres with the aim of systematically collecting data from consecutive BOT patients. In the present analysis, data are presented on women with advanced BOT registered into the MITO14 database and conservatively treated between January 1995 and December 2019.

Methodology The objectives were: i) to evaluate the recurrence rate and to determine predictors of recurrence; ii) to assess the impact of a FSS on disease-free survival (DFS) and disease-specific survival (DSS); iii) to evaluate pregnancy and live birth rates following treatment.

Only patients undergoing FSS and with histologically proven FIGO2014 stage II - III BOTs at final pathology were included. Cases submitted to bilateral salpingo-oophorectomy with uterine preservation were eligible. The following exclusion criteria were considered: i) age $>45$ years; ii) presence of second tumor(s) requiring therapy interfering with the treatment of BOT.

Results A total of 101 patients were recruited. The median follow-up time from primary cytoreduction was 124 months (IQR range 80-177.5). Fifty-five patients (54.5\%) experienced at least one recurrence (median time to first relapse 21 months, IQR range 9-53), 53 of whom (96.3\%) undergoing 
secondary surgery (further FSS in 34). At univariate analysis, significant predictors of relapse were: size of largest extraovarian lesion, peritoneal cancer index, completeness of cytoreduction, type of implants. After multivariable analysis, the size of extra-ovarian lesions and the presence of invasive implants resulted as the only independent predictors of recurrence (Tab. 1). Median DFS and DSS were respectively 96 months (95\% CI, 17.5-174.4) and 290.4 months (95\% CI, 280.8-299.9) (figure 1). Thirty-one patients attempted to conceive: $23(74.2 \%)$ achieved at least one pregnancy and 20 $(64.5 \%)$ gave birth to a healthy child. At the end of the observation period, 96 patients (95\%) showed no evidence of disease, $2(2 \%)$ were alive with disease, and 3 patients (3\%) died from BOT.

Conclusions Despite the recurrence high rate, the survivals and pregnancy outcomes indicate that FSS could be considered in advanced BOTs. Among predictors of recurrence, oophorectomy (vs. cystectomy) has resulted not significant as in earlystage BOTs likely due to the advanced-stage setting. Size of extra-ovarian lesions and presence of invasive implants were the only significant predictors. Completeness of cytoreduction was lacking significance likely because of low number of patients with residual disease.

Disclosures All authors declare no financial support or relationships that may pose conflict of interest.

\section{CHEMOTHERAPY DURING PREGNANCY AND NEONATAL OUTCOME: A RETROSPECTIVE ANALYSIS ON 47 PATIENTS}

${ }^{1}$ Eugenia DI Loreto, ${ }^{2}$ Alessandra Familiari, ${ }^{2}$ Veronica Accurti, ${ }^{3}$ Tiziana Boggini, ${ }^{1}$ Carlotta Castellani, 'Gianpiero Polverino, ${ }^{4}$ Julia Bewart, ${ }^{3}$ Monica Fumagalli, ${ }^{4}$ Fedro Alessandro Peccatori, 'Giovanna Scarfone. 'Fondazione Irccs Ca' Granda - Ospedale Maggiore Policlinico; Department of Gynecology; ${ }^{2}$ Fondazione Irccs Ca' Granda - Ospedale Maggiore Policlinico; Department of Maternal-Fetal Medicine; ${ }^{3}$ Fondazione Irces Ca' Granda - Ospedale Maggiore Policlinico; Neonatal Intensive Care Unit; ${ }^{4}$ European Institute of Oncology (leo); Department of Gynecology

\subsection{6/ijgc-2020-ESG0.85}

Introduction/Background The maternal diagnosis of cancer complicates approximately $0,1 \%$ of all pregnancies. The most frequently diagnosed malignancies are breast cancer, cervical cancer, lymphoma, ovarian cancer and melanoma. Although chemotherapy can be administered during pregnancy, its effects on obstetric and neonatal outcomes are still largely unknown. The aim of this study is to assess the oncologic management as well as the obstetric and perinatal outcomes in a consecutive series of patients diagnosed with cancer during pregnancy. Methodology Retrospective cohort study including 47 pregnant women diagnosed with primary invasive cancer during pregnancy between 2010 and 2019 at IRCCS Policlinico di Milano and Istituto Europeo Di Oncologia (Milan, Italy). All the included patients have been treated with chemotherapy during pregnancy. Oncologic, obstetric and neonatal data have been collected and compared. Linear regression analysis was used to assess the correlation between the therapy and perinatal outcomes. All the analysis were performed with Stat Direct 2.7.9 (StatsDirect Ltd, Altrincham).

Results The most common malignancies diagnosed in our cohort of 47 women were: breast cancer as the most common type $(87,2 \%)$, followed by lymphomas $(6,4 \%)$. All the maternal characteristics are listed in table 1.
Abstract 389 Table 1 Baseline demographics of the study population

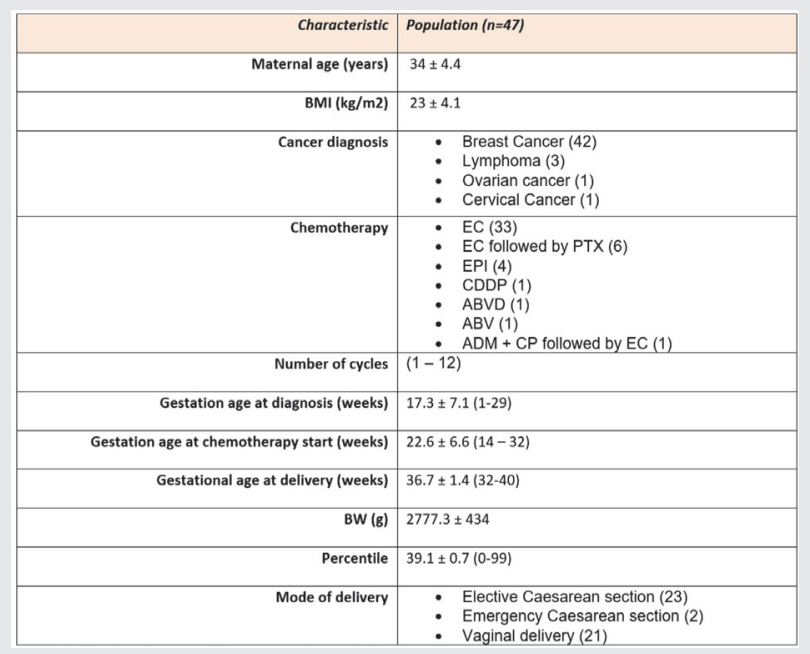

Data are given as mean \pm SD or $\mathrm{n}(\%)$.

$\mathrm{BW}=$ Birthweight, $\mathrm{EC}=$ Epirubicin and cyclophosphamide, PTX=Paclitaxel, CDDP=Cisplatin $C P=$ Cyclophosphamide, $A D M=A d r i a m y c i n, A B V D=A d r i a m y c i n, B l e o m y c i n, D a c a r b a z i n e$, Vinblastine

Abstract 389 Table 2 Results of the linear regression analysis of factors associated with neonatal outcomes

\begin{tabular}{|l|l|l|l|l|l|}
\hline & $\begin{array}{l}\text { BW percentile } \\
P \text { value }\end{array}$ & $\begin{array}{l}\text { 1st minute Apgar } \\
\text { P value }\end{array}$ & $\begin{array}{l}\text { 5th minute Apgar } \\
P \text { value }\end{array}$ & $\begin{array}{l}\text { Neonatal Hb } \\
P \text { value }\end{array}$ & $\begin{array}{l}\text { Neonatal WBC } \\
P \text { value }\end{array}$ \\
\hline$N^{\circ}$ of cycles & 0.63 & 0.28 & 0.18 & 0.88 & 0.77 \\
\hline GA at start & 0.90 & 0.30 & 0.62 & 0.75 & 0.83 \\
\hline
\end{tabular}

BW=Birthweight; $\mathrm{Hb}=$ Haemoglobin, $\mathrm{WBC}=$ White Blood Cells

All the patients were treated with chemotherapy during pregnancy consisting of different number of cycles according to the gestational age at diagnosis $(1-12)$. Maternal chemotherapy-related toxicity was generally lower than expected, maximum grade 1 according to National Cancer Institute Common toxicity Criteria (NCI-CTC).

All pregnancies ended with a livebirth fetus, at a mean gestational age of 36,7 weeks. The delivery was planned at least 3 weeks after the last administration of chemotherapy: 21 patients had a vaginal delivery, 23 an elective caesarean section and 2 an emergency caesarean section.

Table 2 shows the results of the correlation between maternal chemotherapy and perinatal outcomes: neonatal birthweight percentile, Apgar score and blood count parameters. No correlation has been demonstrated neither for the number of cycles or the gestational age at diagnosis and any of the outcome considered.

Conclusion Our results show that administration of chemotherapy during pregnancy is not associated with perinatal complications. Neonatal birth weight, Apgar score and neonatal hematologic indices are not affected by the number of chemotherapy cycles and the gestational age at the beginning of the treatment.

Disclosures Nothing to disclose. 